\title{
Fast, Iterative Image Reconstruction for MRI in the Presence of Field Inhomogeneities
}

\author{
Bradley P. Sutton*, Student Member, IEEE, Douglas C. Noll, Member, IEEE, and \\ Jeffrey A. Fessler, Senior Member, IEEE
}

\begin{abstract}
In magnetic resonance imaging, magnetic field inhomogeneities cause distortions in images that are reconstructed by conventional fast Fourier trasform (FFT) methods. Several noniterative image reconstruction methods are used currently to compensate for field inhomogeneities, but these methods assume that the field map that characterizes the off-resonance frequencies is spatially smooth. Recently, iterative methods have been proposed that can circumvent this assumption and provide improved compensation for off-resonance effects. However, straightforward implementations of such iterative methods suffer from inconveniently long computation times. This paper describes a tool for accelerating iterative reconstruction of field-corrected MR images: a novel time-segmented approximation to the MR signal equation. We use a min-max formulation to derive the temporal interpolator. Speedups of around 60 were achieved by combining this temporal interpolator with a nonuniform fast Fourier transform with normalized root mean squared approximation errors of $0.07 \%$. The proposed method provides fast, accurate, field-corrected image reconstruction even when the field map is not smooth.
\end{abstract}

Index Terms-Field inhomogeneity correction, image reconstruction, iterative methods, magnetic resonance imaging, temporal interpolation, time segmentation.

\section{INTRODUCTION}

D IFFERENCES in the magnetic susceptibility of adjacent regions within an object, which occur for example near air/tissue interfaces in the brain, cause image distortions in magnetic resonance (MR) images formed by conventional reconstruction methods. In spin-warp imaging, off-resonance effects cause spatial shifts and intensity variations [1], whereas spatial blur is induced in noncartesian k-space MR imaging (MRI) (using spirals, etc.) [2]. Many image reconstruction methods have been proposed to correct for the field distortions [3]-[7]. We focus on algorithms appropriate for conventional computers; optical implementations may also be feasible [8]. There are two components to most methods for field-corrected MR image reconstruction. The first procedure is to obtain an estimate of the field map that quantifies the spatial distribution of magnetic field inhomogeneities. The second procedure is to use that field map

\footnotetext{
Manuscript received June 14, 2002; revised October 18, 2002. This work was supported in part by a Whitaker Foundation Graduate Fellowship and in part by the UM Center for Biomedical Engineering Research. The Associate Editor responsible for coordinating the review of this paper and recommending its publication was X. Hu. Asterisk indicates corresponding author.

*B. Sutton is with the Department of Biomedical Engineering, University of Michigan, Ann Arbor, MI 48109-2108, USA (e-mail: bpsutton@umich.edu).

D. C. Noll and J. A. Fessler are with the Department of Biomedical Engineering, University of Michigan, Ann Arbor, MI 48109-2108 USA.

Digital Object Identifier 10.1109/TMI.2002.808360
}

to form a reconstructed image of the transverse magnetization. This paper focuses on the second procedure; like many methods, we assume that an accurate, spatially undistorted field map is available. This simplification underlies most of the field-corrected MR image reconstruction methods. However, in many cases it may be necessary or desirable to couple the field-map estimation and image reconstruction procedures. In such cases, the methods described in this paper could be one component of an overall joint estimation procedure [9].

After a field map is obtained, one method of field-corrected image reconstruction, the conjugate phase method [3], [6], [7], seeks to compensate for the phase accrual at each time point due to the off-resonance. This method, like most noniterative methods, relies on the assumption of a smooth field map. Timesegmented and frequency-segmented approximations exist for this method to speed image reconstruction [3], [7]. Recent work has suggested that the failure of the conjugate phase method in regions where the field map is not smooth may be due to incorrect density compensation coefficients. Spatially varying density compensation may be necessary in those cases, restricting the application of methods to speed computation [10]. Iterative reconstruction methods do not require density compensation coefficients and are immune to discussions on how to calculate accurate density coefficients.

Schomberg [6] provides a rigorous analysis of the family of conjugate-phase methods for off-resonance correction of MR images, and concludes that segmented conjugate-phase methods are preferable to SPHERE methods [5], at least for spiral imaging. Therefore, in this paper we focus on comparing our proposed iterative methods to the conjugate-phase method as the de facto standard for noniterative off-resonance correction. Schomberg's analysis assumes existence of a "time map" relating each k-space point to a unique acquisition time. Our proposed iterative methods do not require any such assumption and are, therefore, applicable to self-intersecting k-space trajectories such as rosettes [11]. Nor are any assumptions about regularity of a time map required for iterative methods.

Model-based iterative reconstruction methods have the potential to account for field maps that violate smoothness assumptions. Munger et al. [12] reported that iterative conjugategradient methods based on Fourier reconstructed echo-planar images outperform the conjugate-phase approach. Their sparsified system model is specific to cartesian trajectories like echo-planar, whereas the conjugate gradient (CG) approach considered here is applicable to any trajectory. Man et al. [13] described an iterative algorithm to remove the residual blur left over after conjugate phase reconstruction in regions with 
rapidly varying inhomogeneity. The iterative reconstruction algorithm proposed in [14] was shown to provide significant improvements in image quality over noniterative methods even for field maps with discontinuities. Their method also can be used in an extended form to estimate more accurate field maps. Unlike standard reconstruction schemes that directly map the $\mathrm{k}$-space data to a reconstructed image, (we will call this a back-projector), most iterative reconstruction methods require a forward-projector (given an estimate of the object and field map, form k-space data) as well as the adjoint of the forward projector.

Interest in iterative reconstruction methods has increased recently due to its utility in multiple coil noncartesian $\mathrm{k}$-space sensitivity encoding (SENSE) problems [15]. Due to the complex aliasing pattern associated with undersampling k-space trajectories such as spirals, iterative methods that include coil sensitivity patterns in the projectors are necessary to reconstruct artifact-free images in practice [15]. Although this paper will focus on field inhomogeneities, one can also apply iterative image reconstruction methods to compensate for other physical phenomena such as deviations in k-space trajectory and relaxation effects, such as $R_{2}^{*}$ [16].

The principal drawback of iterative reconstruction methods has been computation time, with reported values of computation time/iteration ranging up to eight minutes [14]. Recently, accurate and fast nonuniform fast Fourier transform (NUFFT) methods have been developed [17]-[19] and these methods have been applied to MRI data with spiral k-space trajectories [20], [21]. The MR reconstruction problem is closely related to the problem of reconstructing a band-limited signal from nonuniform samples. Strohmer argued compellingly for using trigonometric polynomials (complex exponentials) for finite-dimensional approximations in such problems, and proposed to use an iterative $\mathrm{CG}$ reconstruction method with the NUFFT approach of [22] at its core [23], [24]. In the MR context, this is essentially equivalent to the finite basis expansion we use in (3). In [25], an NUFFT-like algorithm, referred to as "reverse gridding," was applied in combination with the CG algorithm to speed up SENSE image reconstructions. These NUFFT methods have reduced the computation time/iteration to that of noniterative reconstruction methods.

However, the standard NUFFT method by itself does not allow for the compensation of field inhomogeneity effects because the integral signal equation for MR is not a Fourier transform when field inhomogeneities are included. This paper describes several tools for accelerating iterative reconstruction of field-corrected images. Inspired by the time-segmented conjugate-phase reconstruction approach [3], we propose a fast time-segmented forward projector, and its adjoint, that accounts for field effects and uses the NUFFT. The possibility of combining "conventionally used [time or frequency] segmentation approaches" with NUFFT-type methods to correct for field inhomogeneities was noted by Pruessman et al. [25]. However, as we show in this paper, the conventional temporal interpolators (linear, Hanning, etc.) are signficantly suboptimal since they fail to capture the oscillatory nature of phase modulations caused by off-resonance effects. Instead, in this paper we present a temporal interpolation method that is optimal in the min-max sense of minimizing worst-case interpolation error, and compare its accuracy with the "conventional" temporal interpolators. We show that accurate temporal interpolation combined with the NUFFT results in a fast, accurate iterative reconstruction algorithm for field-corrected imaging. We evaluate the accuracy of our time-segmentation interpolator by comparing it to the result of the exact (but slow) evaluation of the signal equation.

This paper starts with an introduction to iterative image reconstruction for MRI in Section II, then we present the derivation of our min-max temporal interpolator for time segmentation in Section II-A. Section II-B describes various ways to compute the interpolator. Section II-C examines the effect of the initial image and preconditioning on the image reconstruction. Simulation and human data experiments are described in Section III with the results given in Section IV.

\section{THEORY}

In MRI, ignoring relaxation effects, the signal equation is given by [26]

$$
s(t)=\int \tilde{f}(\boldsymbol{r}) c(\boldsymbol{r}) e^{-i \omega(\boldsymbol{r})\left(t+T_{E}\right)} e^{-i 2 \pi(\boldsymbol{k}(t) \cdot \boldsymbol{r})} d \boldsymbol{r}
$$

where $s(t)$ is the complex baseband signal at time $t$ during the readout, $T_{E}$ is the echo time, $\tilde{f}(\boldsymbol{r})$ is a continuous function of the object's transverse magnetization at location $\boldsymbol{r}$ immediately following the spin preparation step, $c(\boldsymbol{r})$ is the sensitivity map of the receiver coil, $\omega(\boldsymbol{r})$ is the field inhomogeneity present at $\boldsymbol{r}$, and $\boldsymbol{k}(t)$ is the k-space trajectory. For convenience, we let $f(\boldsymbol{r})=\tilde{f}(\boldsymbol{r}) c(\boldsymbol{r}) e^{-i \omega(\boldsymbol{r}) T_{E}}$. Accurate estimation of $f(\boldsymbol{r})$ yields $\tilde{f}(\boldsymbol{r})$ assuming the sensitivity and field maps are known. In an MR scan, the raw measurements are noisy samples of the signal in (1)

$$
y_{i}=s\left(t_{i}\right)+\varepsilon_{i}, \quad i=1, \ldots, M
$$

where the $\varepsilon_{i}$ 's denote complex Gaussian noise. From these samples we would like to reconstruct $f(\boldsymbol{r})$. The conventional approach for image reconstruction is to interpolate the $y_{i}$ 's onto a cartesian grid in spatial frequency space, after applying sample density compensation, and to then use an inverse FFT and deapodization to estimate samples of $f(\boldsymbol{r})$ [27]. This gridding method, when combined with time segmentation of the field inhomogeneity effects, is a fast conjugate phase approach [3].

The combination of (1) and (2) form a continuous-to-discrete (CD) mapping. This is clearly an ill-posed problem since there is an infinite collection of solutions, $f(\boldsymbol{r})$, that exactly match the data $\boldsymbol{y}=\left(y_{1}, \ldots, y_{M}\right)$. In [28], the pseudoinverse of this CD mapping was investigated for minimum-norm least-squares image reconstruction without field-correction. Although their approach was computationally intensive, the pseudoinverse calculation was object-independent and could be performed once for a given trajectory. However, in the case of field-corrected imaging, the CD mapping is object-dependent because of the specific field map of the slice of interest. This prohibits precalculation of the singular-value decomposition of the CD operator, so we seek more practical methods. 
Instead of finding the pseudoinverse of the CD mapping, we restrict the number of unknowns to be estimated by parameterizing the object and field map in terms of basis functions, $\phi(r)$, assuming that

$$
\begin{aligned}
& f(\boldsymbol{r}) \approx \sum_{n=0}^{N-1} f_{n} \phi_{1}\left(\boldsymbol{r}-\boldsymbol{r}_{n}\right) \\
& \omega(\boldsymbol{r}) \approx \sum_{n=0}^{N-1} \omega_{n} \phi_{2}\left(\boldsymbol{r}-\boldsymbol{r}_{n}\right) .
\end{aligned}
$$

For this paper, we will use the voxel indicator function $\phi_{1}(\boldsymbol{r})=$ $\phi_{2}(\boldsymbol{r})=\operatorname{rect}\left(r_{1} / \Delta_{1}\right) \cdots \operatorname{rect}\left(r_{P} / \Delta_{P}\right)$ for the $P$-dimensional problem. This choice is somewhat natural for display devices that use square areas of nearly constant luminance. However, this parameterization does not model within-voxel field gradients. Regardless of what basis one chooses, (3) is only an approximation and we plan to explore other choices, such as triangle functions, in our future work. Triangle basis functions would allow us to model first-order gradients of the field map and voxel intensities, which may help reduce within-voxel susceptibility effects. Substituting (3) in (1) yields

$$
s(t) \approx \Phi(\boldsymbol{k}(t)) \sum_{n=0}^{N-1} f_{n} e^{-i \omega_{n} t} e^{-i 2 \pi\left(\boldsymbol{k}(t) \cdot \boldsymbol{r}_{n}\right)}
$$

where $\Phi(\boldsymbol{u})$ denotes the Fourier Transform of $\phi(\boldsymbol{r})$. We express the noisy measured samples of this signal in matrix-vector form as follows:

$$
\boldsymbol{y}=\boldsymbol{A f}+\varepsilon
$$

where $\boldsymbol{f}=\left(f_{0}, \ldots, f_{N-1}\right)$ and the elements of the $M \times N$ matrix $\boldsymbol{A}$ are

$$
a_{m, n}=\Phi\left(\boldsymbol{k}\left(t_{m}\right)\right) e^{-i \omega_{n} t_{m}} e^{-i 2 \pi \boldsymbol{k}\left(t_{m}\right) \cdot \boldsymbol{r}_{n}} .
$$

In the discrete-to-discrete formulation (5), our goal is to estimate the image $f$ from the $\mathrm{k}$-space data $y$, accounting for the statistics of the noise $\varepsilon$. This will still be an ill-posed problem if $N>M$, and is usually ill-conditioned even if $N \leq M$ for noncartesian trajectories.

Since the dominant noise in MRI is white Gaussian [29], we estimate $\boldsymbol{f}$ by minimizing the following penalized least-squares cost function

$$
\begin{aligned}
\Psi(\boldsymbol{f}) & =\frac{1}{2}\|\boldsymbol{y}-\boldsymbol{A f}\|^{2}+\beta R(\boldsymbol{f}) \text { so that, } \\
\hat{\boldsymbol{f}} & =\arg \min _{\boldsymbol{f}} \Psi(\boldsymbol{f}) .
\end{aligned}
$$

The second term in the equation for $\Psi(\boldsymbol{f})$ is a regularization function, $R(\boldsymbol{f})$, that penalizes the roughness of the estimated image. This regularization can decrease the condition number of the image reconstruction problem and, therefore, speed convergence. We choose the parameter $\beta$ by examining the point spread function (PSF) of the reconstructed image [30], preferably by choosing $\beta$ small enough to not significantly degrade the spatial resolution relative to the natural resolution associated with the k-space trajectory.

The least-squares cost function used here is appropriate for Gaussian measurement noise. If non-Gaussian error "spikes" are present, then one could use a nonquadratic cost function to provide robustness to those outliers [31], at the expense of increased computation. Alternatively, one could use other methods to detect those spikes, e.g., [32], then exclude the corresponding measurement samples from the iterative reconstruction process; no "interpolation" of samples is needed.

We apply the iterative CG algorithm for minimization of (7). The algorithm is given below for reference. For simplicity, we have used quadratic regularization: $R(\boldsymbol{f})=1 / 2\|\boldsymbol{C} \boldsymbol{f}\|^{2}$ for a matrix $\boldsymbol{C}$ that takes differences between neighboring pixels. The algorithm may also include a data weighting matrix $\boldsymbol{W}$ for performing weighted least squares, i.e., replace $\|\cdot\|^{2}$ with $\|\cdot\|_{W}^{2}$ in (7). One can also include a preconditioning matrix $M$ to speed convergence of the $\mathrm{CG}$ algorithm. Section II-C discusses the weighting and preconditioner matrices in more detail. In the algorithm below, $\boldsymbol{g}_{\text {new }}$ denotes the negative gradient of $\Psi(\boldsymbol{f})$ from (7), $\boldsymbol{r}$ is the residual, $\boldsymbol{d}$ denotes the step direction, and $\alpha$ denotes the step size. The algorithm is started with an initial estimate of the image, $\boldsymbol{f}=\boldsymbol{f}_{0}$. Section II-C discusses the choice of this initial estimate.

$$
\begin{aligned}
& \text { CG Algorithm } \\
& \text { Initialize } \\
& \boldsymbol{r}=\boldsymbol{y}-\boldsymbol{A f}_{0} \quad \text { (residual) } \\
& \text { Iteration Steps } \\
& \boldsymbol{g}_{\text {new }}=\boldsymbol{A}^{*} \boldsymbol{W r}-\beta \boldsymbol{C}^{*} \boldsymbol{C} \boldsymbol{f}_{n} \\
& \gamma= \begin{cases}0, & \text { 1st iteration } \\
\frac{\boldsymbol{g}_{\text {new }} * \boldsymbol{M} \boldsymbol{g}_{\text {new }}}{\boldsymbol{g}_{\text {old }} * \boldsymbol{M} \boldsymbol{g}_{\text {old }}}, & \text { else }\end{cases} \\
& \boldsymbol{d}:=\boldsymbol{M} \boldsymbol{g}_{\text {new }}+\gamma \boldsymbol{d} \\
& q=A d \\
& \alpha=\frac{\boldsymbol{d}^{*} \boldsymbol{g}_{\text {new }}}{\boldsymbol{q}^{*} \boldsymbol{W} \boldsymbol{q}+\beta \boldsymbol{d}^{*} \boldsymbol{C}^{*} \boldsymbol{C d}} \\
& \boldsymbol{f}_{n+1}=\boldsymbol{f}_{n}+\alpha \boldsymbol{d} \text { (update image) } \\
& \boldsymbol{r}:=\boldsymbol{r}-\alpha \boldsymbol{q} \text { (update residual) } \\
& \boldsymbol{g}_{\text {old }}=\boldsymbol{g}_{\text {new }} \text {. }
\end{aligned}
$$

The dominant computation in each iteration of the CG algorithm is computing $\boldsymbol{A d}$ and $\boldsymbol{A}^{*} \boldsymbol{r}$, where the superscript * denotes complex conjugate transpose. Computing $\boldsymbol{A} \boldsymbol{f}$ corresponds to evaluating (4). For cartesian k-space trajectories, one can evaluate (4) quickly via the FFT if the field inhomogeneity is ignored. However, for noncartesian k-space trajectories (spirals, etc.) direct evaluation of (4) is very time consuming. When field inhomogeneity is ignored, a NUFFT [17], [19] can be used to rapidly and accurately evaluate the discrete signal (4) even for noncartesian trajectories. However, the NUFFT method is not directly applicable when the field inhomogeneity is included because (1) is not a Fourier transform integral. We propose to combine the NUFFT and a version of time segmentation [3] (but with min-max temporal interpolation) to compute (4) rapidly and accurately. We first derive the min-max interpolator and then discuss some approaches to computing it. This section concludes with a discussion of proposed methods to speed convergence of the $\mathrm{CG}$ algorithm for iterative MR imaging.

\section{A. Time Segmentation}

In (4), the problem is in the term $e^{-i \omega_{n} t}$, where $t$ is not a constant. If $t$ were a constant, then the term $e^{-i \omega_{n} t}$ could be absorbed into $f_{n}$ and (4) could be evaluated quickly by the NUFFT. The idea of "time segmentation" is to use small time 
segments over which $t$ is approximately constant [3]. For a timesegmented approximation of the term $e^{-i \omega_{n} t}$, we partition the acquisition window into $L$ time segments of width $\tau$ and compute the term at the $L+1$ break points. We then interpolate between these break points to evaluate an approximation at intermediate time points as follows:

$$
e^{-i \omega_{n} t} \approx \sum_{l=0}^{L} a_{l}(t) e^{-i \omega_{n} \tau l}
$$

where $a_{l}(t)$ is the interpolation coefficient for the $l$ th break point for time $t$. Replacing the term $e^{-i \omega_{n} t}$ in (4) with its time-segmented approximation (8) gives

$$
\hat{s}(t)=\Phi(\boldsymbol{k}(t)) \sum_{l=0}^{L} a_{l}(t) \sum_{n=0}^{N-1}\left[f_{n} e^{-i \omega_{n} \tau l}\right] e^{-i 2 \pi\left(\boldsymbol{k}(t) \cdot \boldsymbol{r}_{n}\right)} .
$$

The key property of (9) is that it is a weighted sum of discrete-space Fourier transforms of the term in brackets, weighted by the coefficients $\boldsymbol{a}(t)=\left(a_{0}(t), \ldots, a_{L}(t)\right)^{\prime}$. We can perform these inner FTs quickly and accurately using an NUFFT [19]. Our goal here is to choose the $\boldsymbol{a}(t)$ to minimize the error of approximation (9). In the spirit of [18] and [19], we propose to adopt a min-max criterion to optimize the temporal interpolation coefficients, $\boldsymbol{a}\left(t_{i}\right)$ for $i=1, \ldots, M$, i.e., for every point in the k-space readout. For any time $t$, we choose the coefficients $a(t)$ using the following criterion:

$$
\min _{\boldsymbol{a}(t)} \max _{\boldsymbol{f} \in \mathbb{C}^{N}:\|\boldsymbol{f}\|=1}\left|\frac{\hat{s}(t)-s(t)}{\Phi(\boldsymbol{k}(t))}\right| .
$$

That is, we seek the interpolation coefficients $\boldsymbol{a}(t)$ that will minimize ( $\mathrm{min}$ ) the interpolation error for the object vector, $\boldsymbol{f}$, that causes the largest (max) error of all possible signals. Note that if $\Phi(\boldsymbol{k}(t))=0$, then the error in the approximation (9) would be zero regardless of the interpolator.

The error in the approximation (9) can be expressed as

$$
\begin{aligned}
\frac{\hat{s}(t)-s(t)}{\Phi(\boldsymbol{k}(t))}= & \sum_{n=0}^{N-1} f_{n} e^{-i 2 \pi\left(\boldsymbol{k}(t) \cdot \boldsymbol{r}_{n}\right)} \\
& \cdot\left[e^{-i \omega_{n} t}-\sum_{l=0}^{L} a_{l}(t) e^{-i \omega_{n} \tau l}\right] \\
= & \sum_{n=0}^{N-1} g_{n}(t) f_{n} e^{-i 2 \pi\left(\boldsymbol{k}(t) \cdot \boldsymbol{r}_{n}\right)}, \\
= & \sqrt{N}\langle\boldsymbol{g}(t), \boldsymbol{q}(t)\rangle
\end{aligned}
$$

where $\boldsymbol{g}(t)=\left(g_{0}, \ldots, g_{N-1}\right), \boldsymbol{q}=\left(q_{0}, \ldots, q_{N-1}\right)$, and

$$
\begin{aligned}
& g_{n}(t)=\frac{1}{\sqrt{N}}\left[e^{-i \omega_{n} t}-\sum_{l=0}^{L} a_{l}(t) e^{-i \omega_{n} \tau l}\right] \\
& q_{n}(t)=f_{n}^{*} e^{i 2 \pi\left(\boldsymbol{k}(t) \cdot \boldsymbol{r}_{n}\right)} .
\end{aligned}
$$

Define $b_{n}(t)=(1 / \sqrt{N}) e^{-i \omega_{n} t}$, and let $\boldsymbol{G}$ be an $N$ by $L+1$ matrix with $\boldsymbol{G}_{n \boldsymbol{l}}=(1 / \sqrt{N}) e^{-i \omega_{n} \tau l}$, then

$$
\boldsymbol{g}(t)=\boldsymbol{b}(t)-\boldsymbol{G a}(t) \text {. }
$$

From (12), $\|\boldsymbol{f}\|=\|\boldsymbol{q}(t)\|$ and $\|\boldsymbol{q}(t)\|$ is independent of time. Therefore, using (11), we can rewrite our min-max estimation problem from (10) as follows:

$$
\min _{\boldsymbol{a}(t)} \max _{\boldsymbol{q} \in \mathbb{C}^{N}:\|\boldsymbol{q}\|=1} \sqrt{N}|\langle\boldsymbol{g}(t), \boldsymbol{q}\rangle| \text {. }
$$

By the Cauchy-Schwarz inequality, for a given time $t$, the worst-case $\boldsymbol{q}$ is $\boldsymbol{g}^{*}(t) /\|\boldsymbol{g}(t)\|$, i.e.,

$$
\max _{\boldsymbol{q} \in \mathbb{C}^{N}:\|\boldsymbol{q}\|=1}|\langle\boldsymbol{g}(t), \boldsymbol{q}\rangle|=\|\boldsymbol{g}(t)\| .
$$

Note that this is the approximation error in (8). Inserting this worst-case $\boldsymbol{q}$ into the min-max criterion (14) and applying (13) reduces the min-max problem to

$$
\min _{\boldsymbol{a}(t)}\|\boldsymbol{b}(t)-\boldsymbol{G a}(t)\| .
$$

The solution to this least-squares problem yields the min-max interpolator

$$
\boldsymbol{a}(t)=\left(\boldsymbol{G}^{*} \boldsymbol{G}\right)^{-1} \boldsymbol{G}^{*} \boldsymbol{b}(t)
$$

where

$$
\begin{aligned}
{\left[\boldsymbol{G}^{*} \boldsymbol{G}\right]_{l, l^{\prime}} } & =\frac{1}{N} \sum_{n=0}^{N-1} e^{-i \omega_{n} \tau\left(l^{\prime}-l\right)} \\
{\left[\boldsymbol{G}^{*} \boldsymbol{b}(t)\right]_{l} } & =\frac{1}{N} \sum_{n=0}^{N-1} e^{-i \omega_{n}(t-\tau l)}
\end{aligned}
$$

for $l, l^{\prime}=0, \ldots, L$. To compute the min-max interpolator, we form the $(L+1) \times(L+1)$ matrix $G^{*} G$ and multiply its inverse by the $(L+1) \times 1$ vector $\boldsymbol{G}^{*} \boldsymbol{b}(t)$. Typically, $L \ll N$ so this is feasible.

\section{B. Computing the Min-Max Interpolator}

The interpolator in (17) is object dependent since it is a function of the field map, $\boldsymbol{\omega}=\left(\omega_{0}, \ldots, \omega_{N-1}\right)$ and, therefore, must be computed after an initial estimate of the field map is formed. To compute $\boldsymbol{G}^{*} \boldsymbol{G}$ efficiently, first form the column sums of $\boldsymbol{G}$ as follows:

$$
\gamma_{l} \triangleq \frac{1}{\sqrt{N}} \sum_{n=0}^{N-1} \boldsymbol{G}_{n, l} .
$$

Then, using (18), we evaluate the elements of $G^{*} G$ as follows:

$$
\left[\boldsymbol{G}^{*} \boldsymbol{G}\right]_{l, l^{\prime}}= \begin{cases}\gamma_{l^{\prime}-l} & l^{\prime}-l \geq 0 \\ \gamma^{*}{ }_{l-l^{\prime}} & \text { otherwise. }\end{cases}
$$

This is a very fast way to compute $G^{*} \boldsymbol{G}$ for the min-max interpolator.

The sums in (18) do not depend on the spatial arrangement of the field map. This independence suggests that we could compute these sums using simply a histogram of the field map values. We have investigated approximating the computation of (18) by forming the histogram of the field map using $N_{B}$ equal-sized bins covering the range of offset frequencies induced by the field inhomogeneity. Let $m_{p}$ be the number of field map values that fall into bin $p$ with a center off-resonant frequency of $f_{p}$. Then, we can approximate (18) by

$$
\begin{aligned}
{\left[\boldsymbol{G}^{*} \boldsymbol{G}\right]_{l, l^{\prime}} } & \approx \frac{1}{N} \sum_{p=1}^{N_{B}} m_{p} e^{-i 2 \pi f_{p} \tau\left(\boldsymbol{l}^{\prime}-l\right)} \\
{\left[\boldsymbol{G}^{*} \boldsymbol{b}(t)\right]_{l} } & \approx \frac{1}{N} \sum_{p=1}^{N_{B}} m_{p} e^{-i 2 \pi f_{p}(t-\tau l)} .
\end{aligned}
$$

We compute (21) efficiently via a FFT of $m_{p}$, since we use equally spaced histogram bins. We call this approach the histogram approximation to the min-max interpolator. This quantization of the field map into a histogram is somewhat akin to 
the frequency-segmentation method for reducing computation in the conjugate-phase approach for field inhomogeneity correction [33], [34].

The expression for this interpolator bears a striking resemblance to the "multifrequency interpolator" proposed by Man et al. [7]. However, the use of the two interpolators is quite different. The multifrequency interpolator is applied to a set of images that have each been reconstructed by a constant demodulation approximation to the conjugate-phase approach for field inhomogeneity correction. In contrast, our min-max interpolator is applied to predicted k-space signals. The multifrequency interpolation approach inherits the fundamental limitations of the conjugate-phase approach (in particular the requirement of a spatially smooth field map) which are illustrated in the figures in Section IV.

The min-max interpolator (17) depends on the field map and should be recomputed if the field map changes. To avoid recalculating the interpolator coefficients when a field map is updated, we also investigated the use of an object-independent histogram for the field map values. A generic histogram for field maps was used to calculate the interpolator coefficients in (21) and we will refer to this approach as the generic histogram approximation. Several shapes and ranges for generic histograms were examined.

\section{Speeding Convergence of the CG Algorithm}

It has been suggested that a weighted-least squares approach be used to speed convergence of the CG algorithm for iterative MR image reconstruction and that the weights be the coefficients of the sampling density compensation function [25]. However, there has been some discussion on how to calculate optimal density compensation factors [35]-[39] and the iterative image reconstruction algorithm does not require and is not dependent upon these weights if an unweighted (i.e., weighting is unity) least squares approach is used instead. Also, assuming the noise in MRI is white Gaussian, using nonuniform weighting would be suboptimal statistically according to the Gauss-Markov Theorem. Using nonuniform weighting may appear to provide faster convergence in the initial steps of the algorithm for some choices of initial image, but would prevent convergence to the minimum variance solution. Although, Pruessmann et al. [25] state that the signal-to-noise ratio (SNR) penalty is negligible when the density compensation function is used as the weights, we will next discuss how to benefit from this approach without risking any SNR.

Consider the $\mathrm{CG}$ algorithm in (8) when an initial estimate of the image of zeros is used: $\boldsymbol{f}_{0}=\mathbf{0}$. Then, the first iteration gives

$$
\boldsymbol{f}_{1}=\alpha \boldsymbol{A}^{*} \boldsymbol{W} \boldsymbol{y} .
$$

If the data weighting matrix $\boldsymbol{W}$ were just the identity matrix, then this first iteration would simply give the conjugate phase reconstruction without density compensation. If $\boldsymbol{W}$ were instead equal to the density compensation factors, then the first iteration yields a density-compensated conjugate phase reconstruction. Therefore, rather than using an inappropriately weighted CG algorithm, we use the conjugate phase image (reconstructed via a fast, density-compensated, time-segmented approach) as the initial estimate, $\boldsymbol{f}_{0}$. As noted in [40], initializing with a good density-compensated conjugate phase image ensures that subsequent iterations will improve on this initial guess.

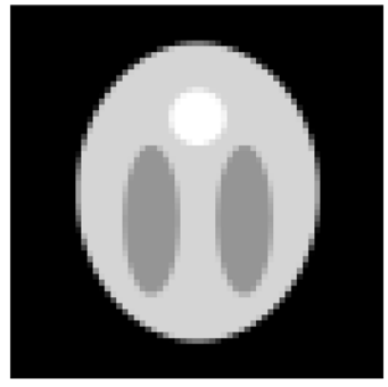

(a)

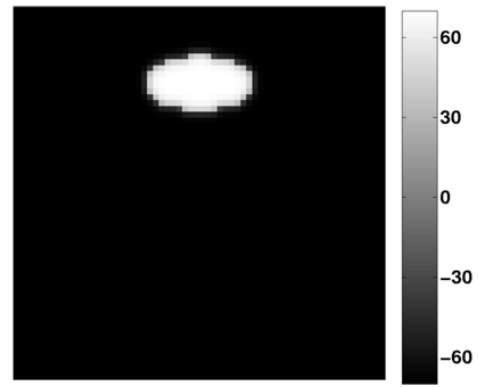

(b)
Fig. 1. (a) Simulation object and (b) field map in Hertz.

Convergence of iterative algorithms can be accelerated by the use of an appropriate preconditioner, e.g., $\boldsymbol{M}$ in (8). Circulant preconditioners have been shown to be effective in shift-invariant problems in tomographic imaging [41]. These preconditioners attempt to undo the blurring induced by applying the forward projector and its adjoint. A circulant preconditioner should be particularly helpful for MR reconstruction with small off-resonance effects, where the PSF is nearly shift invariant, but may also be of some benefit in regions of higher off-resonance effects. Our results to date with circulant preconditioners have shown mild improvements in convergence rate. Preconditioners have also been designed for shift-variant problems [42] and such methods will be investigated for MRI in our future work.

\section{METHODS}

Three sets of studies were performed to evaluate the accuracy and utility of our min-max interpolated iterative reconstruction algorithm. All three studies used a single-shot spiral k-space trajectory with a $T_{E}$ of $25 \mathrm{~ms}$, matrix size of $64 \times 64$, and field of view of $22 \mathrm{~cm} \times 22 \mathrm{~cm}$, giving $3770 \mathrm{k}$-space points. The length of the readout interval was $18.9 \mathrm{~ms}$, so $100-\mathrm{Hz}$ off-resonance causes $3.8 \pi$ extra spin phase accrual during the readout.

\section{A. Interpolator Accuracy}

We performed a simulation study to evaluate the maximum interpolation error, $\|\boldsymbol{g}(t)\|$ in (15), over a finely sampled range of times, $t$, for several temporal interpolators. We used the field map $\boldsymbol{\omega}$ shown in Fig. 1. We observed empirically that, for many field maps, the min-max optimal temporal interpolator could have a significant imaginary component, and this imaginary component contributes to the overall accuracy of the min-max interpolation method. Conventional temporal interpolators used in MRI have been real valued, so to simplify comparisons between the proposed min-max approach and the conventional approaches, we shifted the field map values to a range where the min-max interpolator had a very small imaginary component, as illustrated in Fig. 3. We compared the following interpolation methods: linear interpolation of the two nearest endpoints to the time sample of interest, a Hanning window interpolation using only the two nearest endpoints (similar to that used in [3] for the back-projector problem), the ideal min-max interpolator (17), the histogram approximation to the min-max interpolator calculated using (21) with 1000 bins, and an interpolator using a generic histogram also calculated using (21). Various shapes (flat and triangular) and ranges were used 
for the generic histogram to determine the effect of accuracy of the histogram on the error of the interpolator.

\section{B. Simulation Study}

We performed a series of simulation studies using the same field map and a simulation object shown in Fig. 1. The simulation data was formed by constructing a simulation phantom at a matrix size of $256 \times 256$ and then applying (4) to compute the signal at the desired $\mathrm{k}$-space locations. To avoid intravoxel effects from gradients of the field map inside our larger reconstructed voxels (matrix size of $64 \times 64$ ), we constructed the simulated field map at a size of $64 \times 64$ and zero-order-hold interpolated it up to $256 \times 256$ to create the simulation field map. (We also present one case that includes intravoxel dephasing for comparison). Noise-free simulation studies were conducted to examine the effect of iteration on the interpolation error by computing the normalized root mean squared (NRMS) difference in the reconstructed image of the interpolated, time-segmented approach versus using the exact (slow) signal (4) at convergence.

For the rest of the simulation studies, zero-mean complex Gaussian noise was added to the k-space data to give an SNR of approximately 100, calculated as the ratio of the norms of the $\mathrm{k}$-space data vector and the noise vector: $\|s\| /\|\varepsilon\|$. We examined the normalized root-mean-squared error (NRMSE) between the reconstructed image and the known simulation object. This measure was used to examine accuracy and convergence rate of our proposed iterative algorithm. In the simulation and human studies, the NUFFT was used with the following parameters: two times oversampling, a neighborhood size of $5 \times 5$, and an optimized Kaiser-Bessel window and scaling factors [18].

\section{Human Study}

The time-segmented, NUFFT reconstruction scheme was applied to a human data set collected on a 3.0T GE Signa Scanner in accordance with the Institutional Review Board of the University of Michigan. For the human data, the field inhomogeneity map must be measured by acquiring two gradient echo images with slightly different echo times [43]. To minimize field inhomogeneity distortions in the images used to estimate the field map, we acquired a pair of 4-shot gradient echo images with $T_{E}$ 's of 5 and $7 \mathrm{~ms}$. This fieldmap was used to reconstruct field-corrected images of the same slices with single-shot spirals at a $T_{E}$ of $25 \mathrm{~ms}$. The proposed fast, iterative reconstruction scheme was compared with the conjugate phase method and an uncorrected gridding reconstruction. Since the exact object is not known in a human data set, we attempted to match the full conjugate phase and iterative reconstruction times and qualitatively compare the resulting images.

\section{RESULTS}

\section{A. Interpolator Accuracy}

Fig. 2 shows the maximum interpolation error for $L=1$ through $L=13$ time segments for the five interpolators described in Section III-A. The error given, $\max _{t}\|\boldsymbol{g}(t)\|$, is the maximum error in interpolation as given in (15) over a range of times $t$. The generic histogram used was flat over the range of $[-75,75] \mathrm{Hz}$. The min-max interpolators (ideal min-max, histogram min-max, and generic histogram min-max) have been plotted until the condition number of the $\left(\boldsymbol{G}^{*} \boldsymbol{G}\right)$ matrix becomes

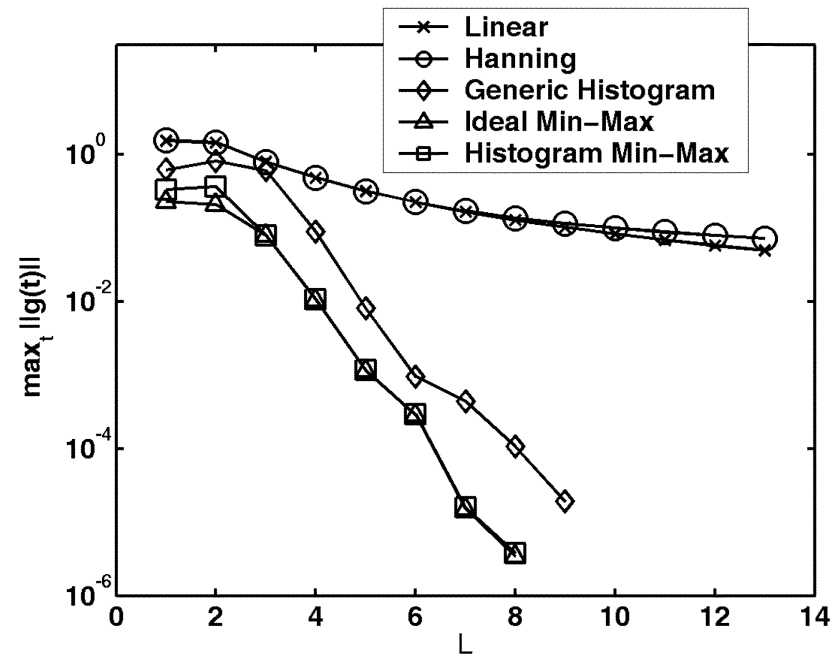

Fig. 2. Maximum interpolation error over a range of time points for each interpolator for various numbers of time segments. Error given is the maximum error in interpolation over a range of times as given in (15).
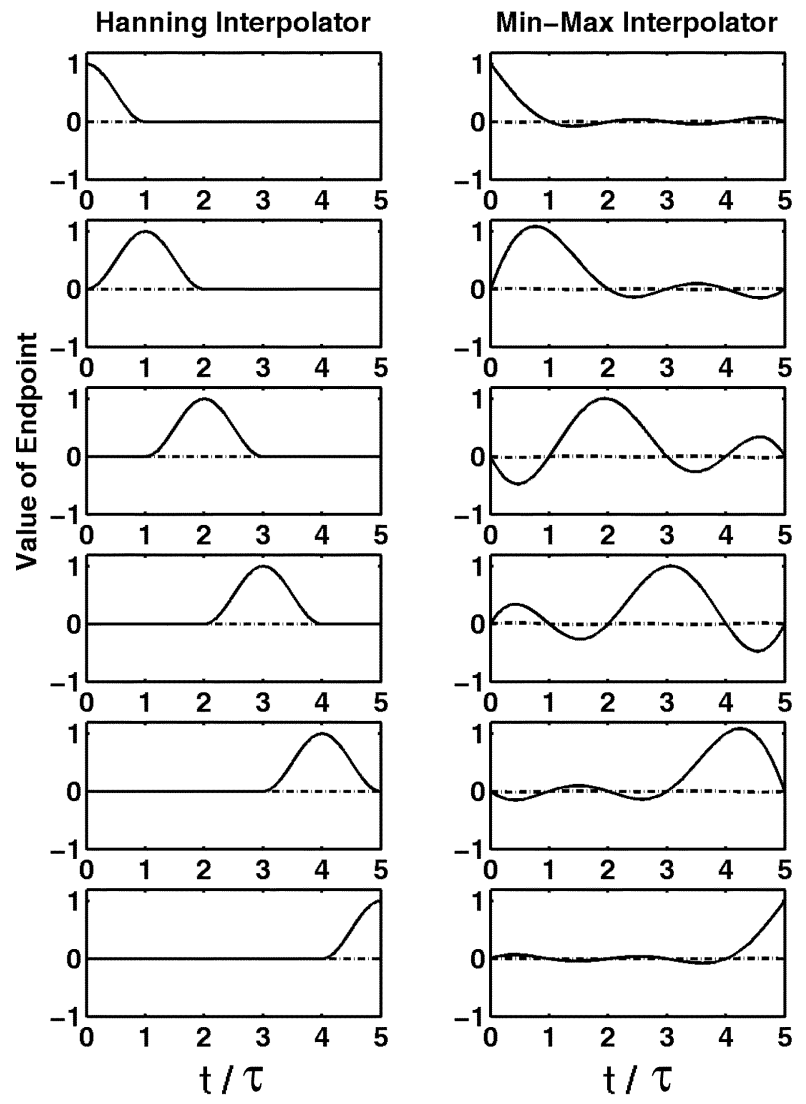

Fig. 3. Real (solid lines) and imaginary (dashed lines) parts of interpolators using $L=5$ for the Hanning and min-max interpolators for the field map given in Fig. 1.

too large for inversion. For $L=8$ the maximum error for the min-max and histogram interpolator is more than four orders of magnitude lower than that of the linear and Hanning "conventional" interpolators.

Fig. 3 shows the Hanning and min-max interpolators for $L=$ 5 . The real and imaginary parts of the min-max interpolator are oscillatory, a property not found in the conventional interpolators. The histogram interpolators looked very similar to the ideal 


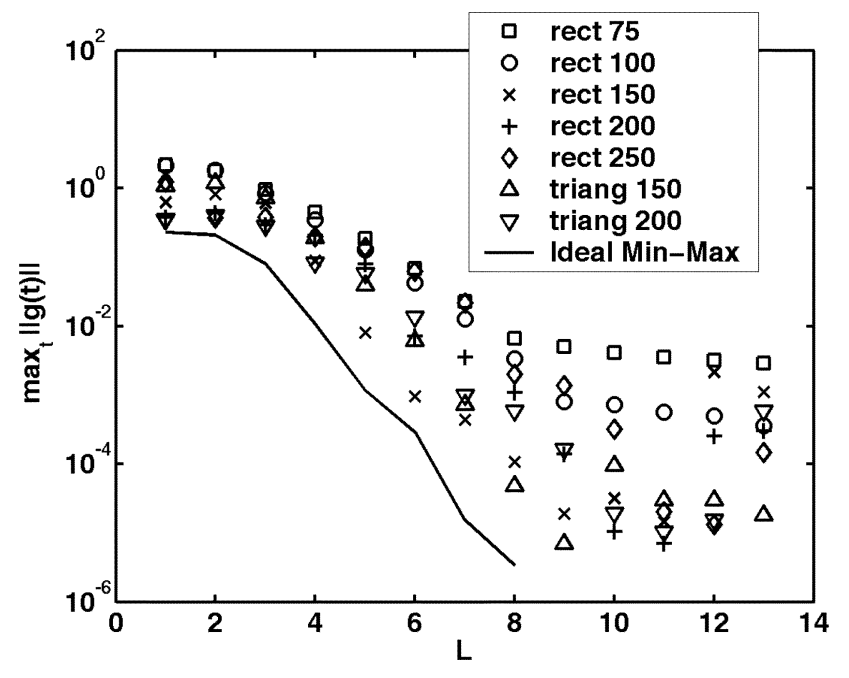

Fig. 4. Comparison of maximum interpolation error of various generic histogram approximate min-max interpolators.

min-max interpolator, even though the generic histogram had a different range of off-resonance frequencies and different histogram shape (flat). Even though it was not explicitly required in our formulation, the min-max interpolators appear to sum to unity at every time point, a property expected of interpolators.

When a histogram of the field map is used that differs from the actual field map (generic histogram), the max error in Fig. 2 showed a slightly higher level of error compared with the ideal min-max interpolator and required a larger number of time segments. We investigated several generic histograms, rectangular and triangular shapes, and several different ranges of off-resonance, $75,100,150,200$, and $250 \mathrm{~Hz}$. All the generic histograms were centered around $0 \mathrm{~Hz}$, to agree with the simulated field map. Fig. 4 shows the maximum NRMSE for various numbers of time segments. As seen in this figure, the interpolator is relatively immune to moderate changes in the histogram of the field map. At an $L$ value of 11, the rectangular histograms with ranges of 150,200 , and $250 \mathrm{~Hz}$ and triangular histograms with ranges of 150 and $200 \mathrm{~Hz}$ all provide maximum interpolation errors below $10^{-4}$. Given the independence on spatial arrangement in the formulation of the ideal min-max interpolator, we need only have a range of off-resonance in our histogram that is similar to that of the exact field map.

\section{B. Simulation Study}

As described in Section II-C, we examined the convergence of the CG algorithm under various conditions using the simulation object and field map shown in Fig. 1. Considering the max error in Fig. 2, we selected $L=6$ to give a low error for the min-max interpolator, and examined the error of time segmentation versus using the exact (slow) signal (4) over iteration to see how the error propagates through the iterative process. Fig. 5 shows the NRMS difference between $\boldsymbol{f}_{k}^{\text {approx }}$ and $f_{100}^{\text {exact }}$ where $f_{k}^{\text {approx }}$ denotes the $k$ th iteration of CG algorithm with the fast approximation (9) using various interpolators and $f_{100}^{\text {exact }}$ denotes the 100th iteration (i.e., essentially at convergence) of CG using the exact (slow) signal (4). As shown in Fig. 5, interpolation errors can cause the CG algorithm to converge to a different image. The linear and Hanning interpolated iterative

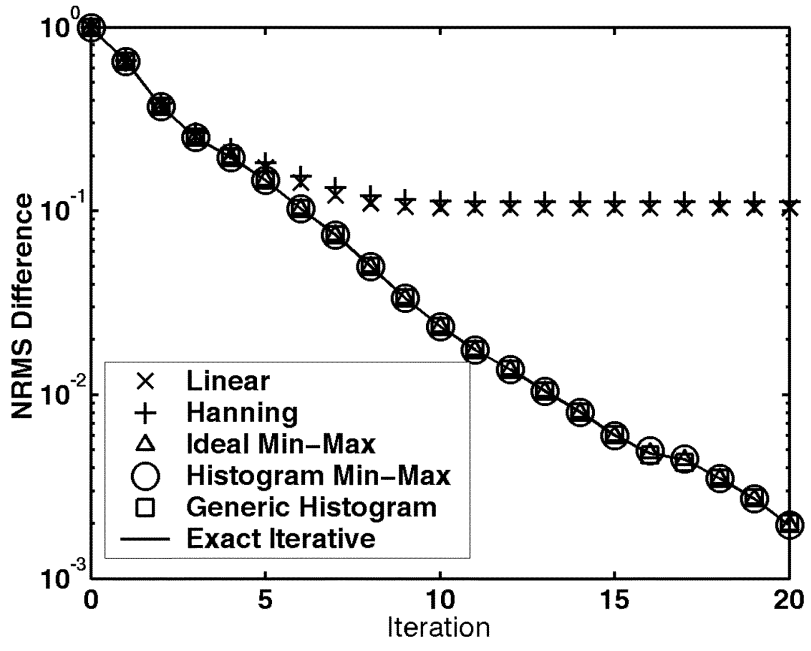

Fig. 5. NRMS difference between $\boldsymbol{f}_{k}^{\text {approx }}$ and $\boldsymbol{f}_{100}^{\text {exact }}$ for $L=6$ in simulation study.

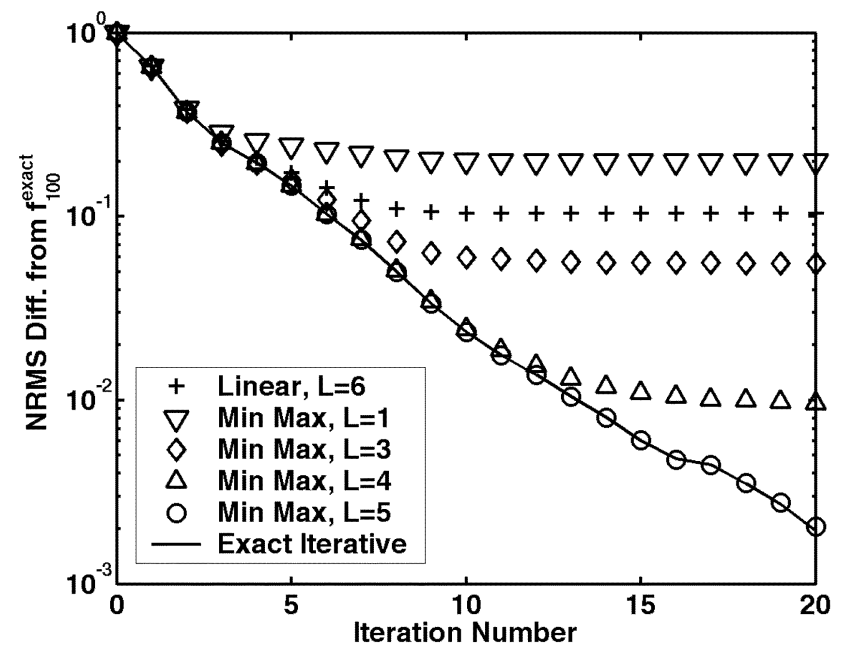

Fig. 6. NRMS difference between $f_{k}^{\text {approx }}$ using the ideal min-max interpolator for $L=1,3,4,5$ and $\boldsymbol{f}_{100}^{\text {exact }}$ over 20 iterations. The time to compute the exact iterative method, using $(4)$, was $\approx 12.7$ s/iteration while the time to compute the fast, interpolated iterative method, using (9), was $(0.019+0.030(L+1))$ s/iteration.

methods converge to a final image that differs from the exact final image by more than $10 \%$ NRMS.

To choose a value for $L$ that gives fast computation yet retains good reconstruction accuracy, we examined the NRMS difference between the interpolated and exact iterative methods for various values of $L$. Fig. 6 shows the NRMS difference between $\boldsymbol{f}_{100}^{\text {exact }}$ and $\boldsymbol{f}_{k}^{\text {approx }}$ over 20 iterations using the ideal min-max interpolator for $L=1,3,4,5$. Computation time for the min-max interpolated iterative method is approximately proportional to $L+1$. On a $2-\mathrm{GHz}$ Xeon workstation using Matlab (The Mathworks, Natick MA), our implementation of the exact (slow) iterative method, using (4), took $\approx 12.7$ s/iteration to evaluate. The min-max interpolation method, took approximately $(0.019+0.030(L+1))$ s/iteration for values of $L=1, \ldots, 13$. The linear interpolated method took approximately the same computation time as the min-max interpolated method and is shown for reference in Fig. 6. Depending on the noise level expected in our reconstructed 
TABLE I

COMPUTATION TIME AND NRMSE BETWEEN $\hat{f}$ AND $f_{\text {true }}$ FOR SimUlation STUdy

\begin{tabular}{l|c|c|c}
\hline Reconstruction Method & Time (s) & NRMSE of complex & NRMSE of magnitude \\
\hline No Correction & 0.06 & 1.35 & 0.22 \\
\hline Full Conjugate Phase & 4.07 & 0.31 & 0.19 \\
\hline Fast Conjugate Phase & 0.33 & 0.32 & 0.19 \\
\hline Fast Iterative (10 iters) & 2.20 & 0.04 & 0.04 \\
\hline Exact Iterative (10 iters) & 128.16 & 0.04 & 0.04 \\
\hline
\end{tabular}

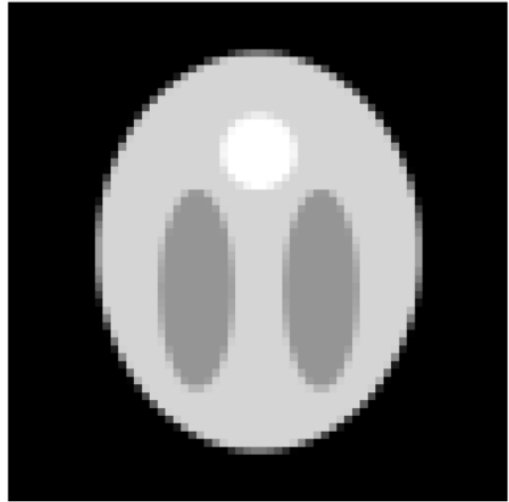

(a)

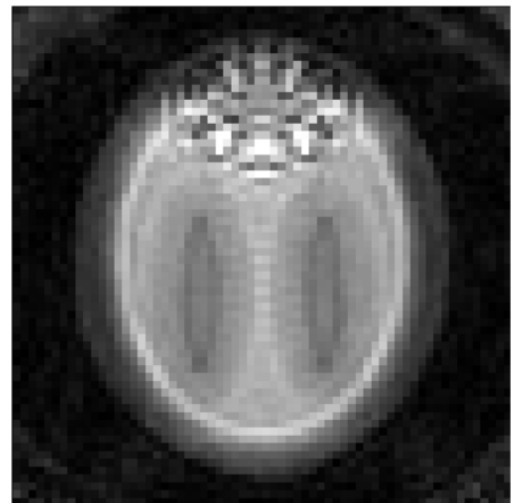

(d)

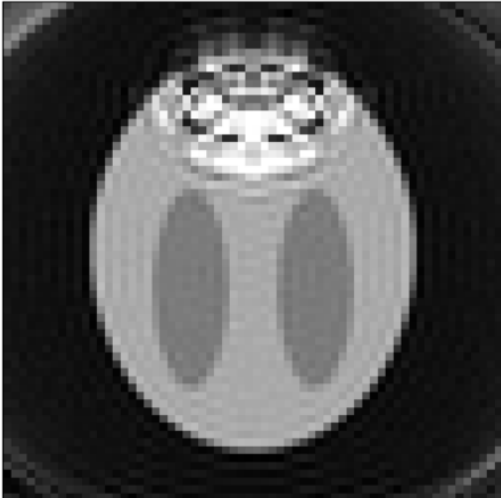

(b)

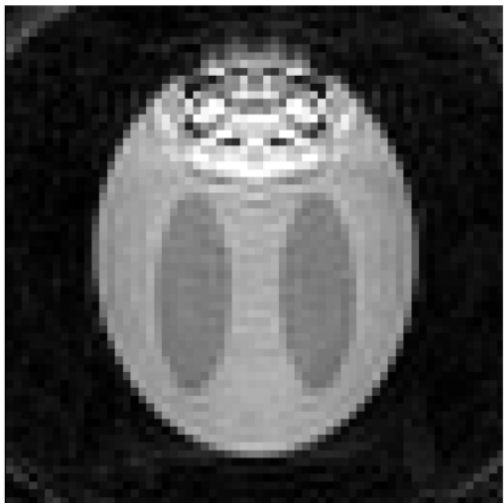

(e)

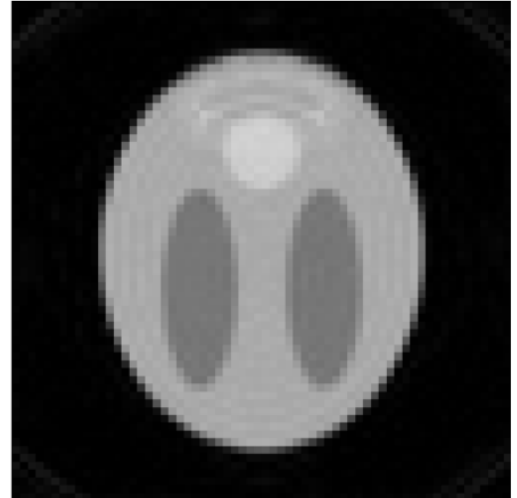

(c)

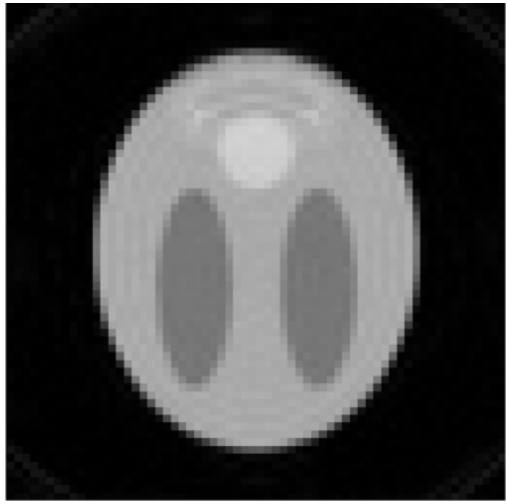

(f)

Fig. 7. Reconstructed images from the simulation study. (a) Simulation object. (b) Slow conjugate phase. (c) Slow iterative. (d) No correction. (e) Fast conjugate phase. (f) Fast iterative.

images, a value of $L=4$ might be reasonable for the min-max interpolator. We chose to use $L=5$ for the ideal min-max interpolator for our simulation and human data studies with a time/iteration of $0.2 \mathrm{~s}$, a speed-up of around 60 over the exact iterative method.

Next, given the exact field map, we ran a simulation study with noise to compare the errors in the reconstructed images under five different reconstruction schemes: no correction for field inhomogeneities, a conjugate-phase reconstruction with density compensation, a fast conjugate phase reconstruction using time segmentation according to [3], the exact (slow) evaluation of the signal equation used in combination with the CG algorithm (the slow iterative method), and the NUFFT with min-max temporal interpolation used in combination with the $\mathrm{CG}$ algorithm (the fast iterative method, $L=5$ ).
The results of NRMSE and computation time are shown in Table I. The NRMSE was calculated over a mask defined by the true object's support. Fig. 7 shows the reconstructed images. The full iterative and fast iterative methods give virtually the same results with a NRMS difference between the two reconstructions of $0.07 \%$, but the fast iterative method takes only $2.2 \mathrm{~s}$ for ten iterations as compared with $128 \mathrm{~s}$ for the slow iterative method. The unsegmented, density-compensated conjugate-phase reconstruction takes $4 \mathrm{~s}$ and both conjugate phase reconstructions produce serious artifacts in regions where the field map is not smoothly varying, and these artifacts propagate to nearby regions.

To verify that interpolator accuracy is important in reconstructing field-corrected images, we compared reconstructions from the CG algorithm using NUFFT with linear, Hanning, and 


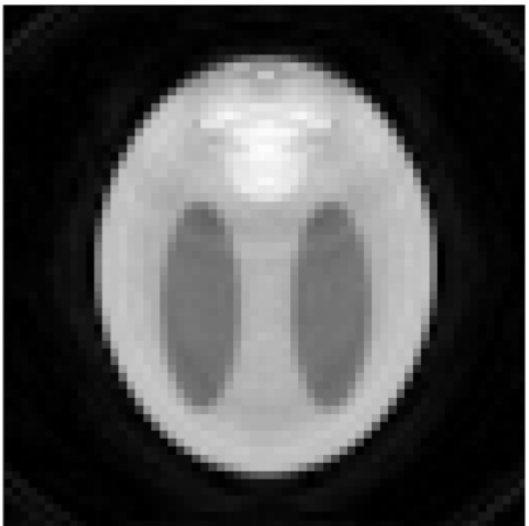

(a)

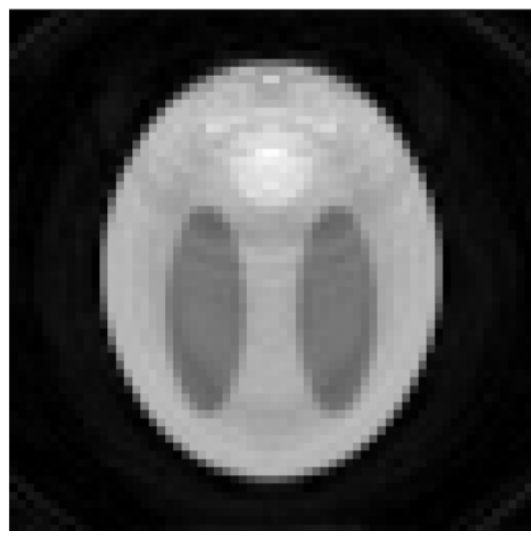

(b)

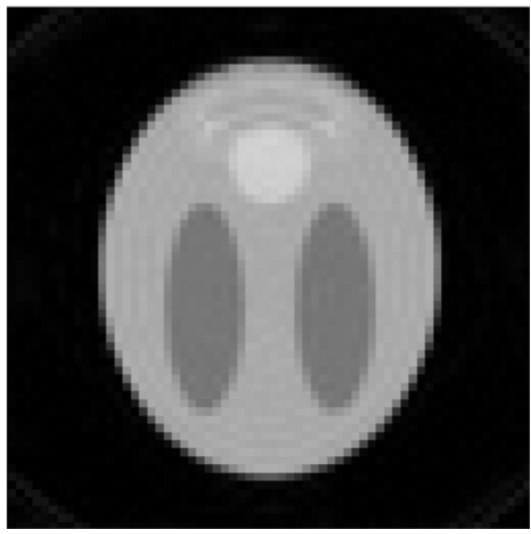

(c)

Fig. 8. Effect of temporal interpolator on fast iterative reconstructions. (a) Linear interpolation. (b) Hanning interpolator. (c) Ideal min-max interpolator.

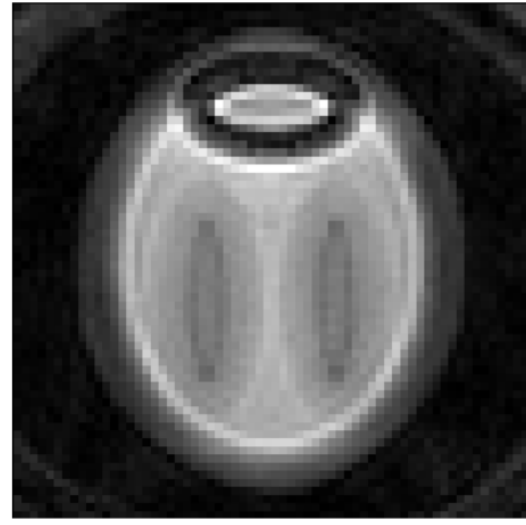

(a)

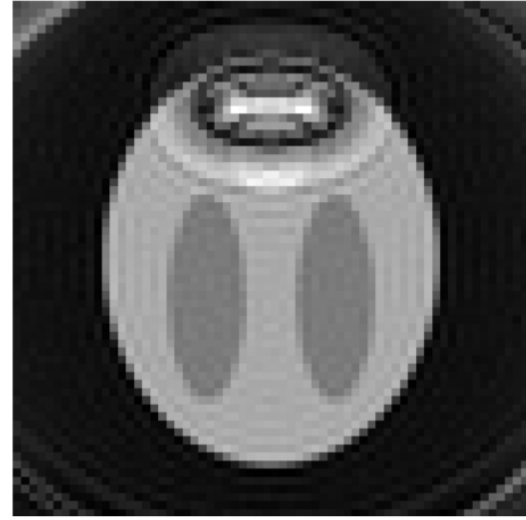

(b)

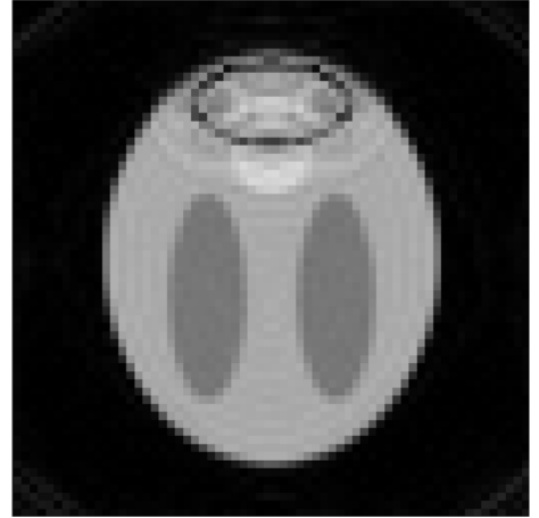

(c)

Fig. 9. Reconstructed images from a simulation study with intravoxel field effects. (a) No correction. (b) Slow conjugate phase. (c) Fast iterative.

ideal min-max intepolators. Fig. 8 shows the reconstructions using $L=5$ and ten iterations of the CG algorithm. The standard interpolators are insufficiently accurate and the algorithm converges to a distorted image, whereas the min-max method yields a nearly undistorted image. This behavior agrees with the quantitative comparison shown in Fig. 5.

As mentioned in Section III, the simulated field map was purposefully constructed to avoid intravoxel dephasing due to within-voxel field inhomogeneities. To show the effects of such dephasing on the field-corrected reconstructions of Fig. 7, we simulated a field map at a $256 \times 256$ matrix size that allowed gradients across the voxels when reconstructed at a matrix size of $64 \times 64$. Fig. 9 shows the reconstructed images. As this figure shows, by assuming basis functions of $\operatorname{rect}(\boldsymbol{r})$, we are unable to model the field gradients across the voxel and the result is signal loss where the field gradient is high. In the iterative reconstruction, this degradation is localized primarily in the pixels where the high gradient occurs. In the conventional field correction, the artifacts are more widespread. We plan to implement triangular basis functions in our future work to model linear intravoxel susceptibility gradients, or to use over-sampled field maps.

\section{Human Data}

As a final comparison, we reconstructed real data collected from a slice of the brain using both the proposed iterative method and a full conjugate phase method. Although the proposed iterative method can be used in an extended form to estimate an undistorted field map, in this case we focused on comparing computation time, so both reconstructions used a field map obtained in the standard way from two short $T_{E}$ (5, $7 \mathrm{~ms})$ 4-shot gradient echo images. For convenience in the iterative method, we used the generic histogram (flat, $[0,150] \mathrm{Hz})$ since it does not depend on the specific field map and can be computed in advance for a given trajectory (depends only on number of time points and a chosen range of off-resonance frequencies). The range of the generic histogram, $[0,150] \mathrm{Hz}$, was chosen to agree with our routinely acquired field maps from the slice of interest. The NUFFT used the parameters given in Section III-B and the min-max interpolator used $L=8$. The reconstruction time for the full conjugate phase was about $4 \mathrm{~s}$, the time for ten iterations of the proposed fast iterative method was $3.6 \mathrm{~s}$. Fig. 10 shows the reconstructed images for two slices. Artifacts in regions of high off-resonance are reduced significantly by the iterative approach. The conjugate phase reconstruction suffers from ringing and piling-up artifacts near the region of field inhomogeneity. Residual signal loss in the iterative reconstruction could be due to a high in-plane gradient in the field map as discussed in Section IV-B, or may be due to through-plane susceptibility gradients. We plan to incorporate models of both phenomena in our future work. Also, the iterative method can be used to 

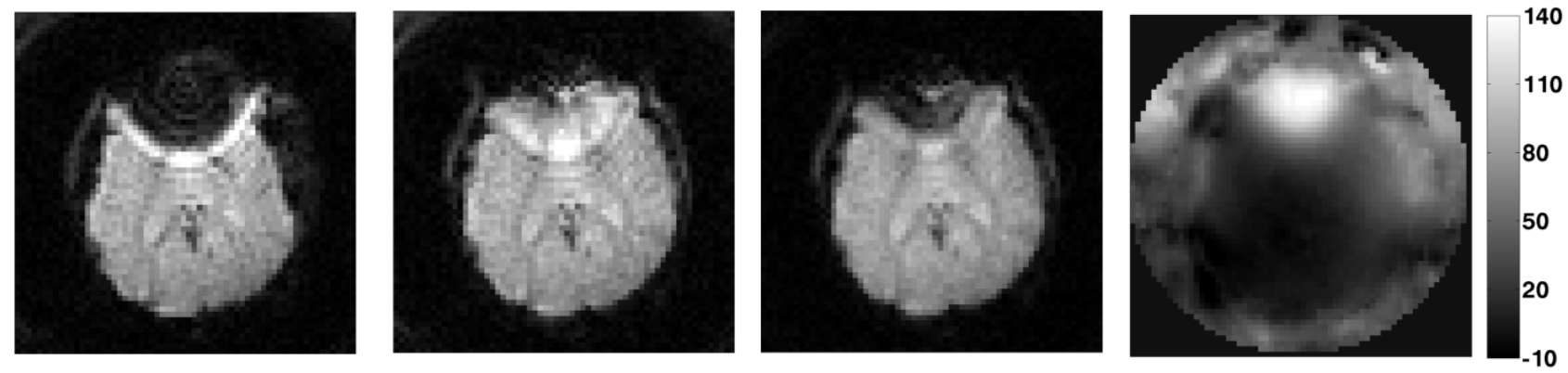

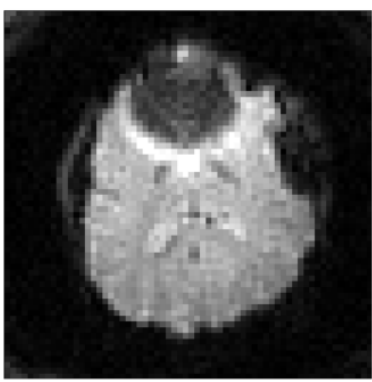

(a)

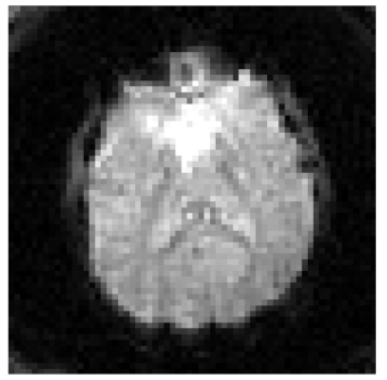

(b)

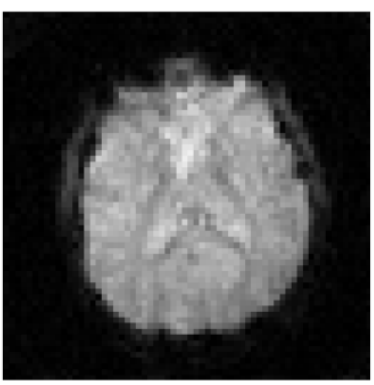

(c)

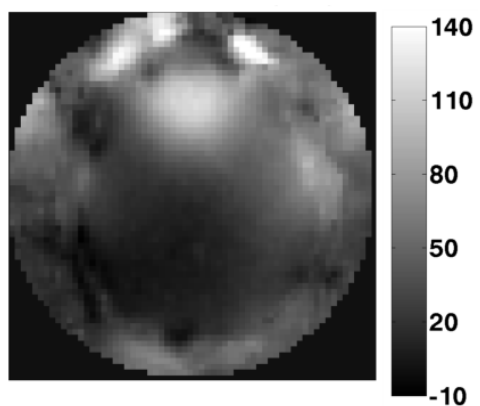

(d)

Fig. 10. Distorted image, its field map, conjugate phase and iterative image reconstructions for two slices. The time for the field-corrected reconstructions were about $4 \mathrm{~s}$ each. (a) Uncorrected. (b) Conjugate phase. (c) Fast iterative. (d) Field map (Hz).

simultaneously estimate an undistorted field map and provide a better field-corrected image [14], [44].

\section{DISCUSSION}

We have presented a method that allows fast, iterative reconstruction of field-corrected MR images. By combining the NUFFT with time segmentation using a min-max temporal interpolator, a computation speed up of a factor of around 60 is achievable with NRMS error in the reconstructed image of $0.07 \%$. We have also developed an approximation to the min-max interpolator that depends on the object-specific field map only through the range of off-resonant frequencies yet provides accuracies near those of the ideal min-max interpolator. For a given trajectory, this interpolator can be precomputed and stored. We have shown that this approximation is relatively robust to small changes in the shape or range of the histogram of the field map. This method should easily be adaptable to other forms of iterative reconstruction in MRI, including SENSE to allow fast, field-corrected SENSE reconstructions [45].

We envision the iterative reconstruction algorithm in the general case to proceed as follows: first, an initial field map is formed via a gridding reconstruction on data at two different echo times. This initial estimate of the field map is used to derive an interpolator for the min-max time interpolation. The estimate of the field map is also used, via a fast conjugate phase reconstruction, to give an initial estimate to the iterative reconstruction. The iterative reconstruction is then run in extended mode with simultaneous estimation of field map and image either by explicit joint estimation [44] or by alternating updates [6], [14]. After several loops of updating the image and field map, we are left with an undistorted estimate of the image and field map.
If the field map has a strong linear component, then it may be possible to adapt the method of Irarrazabal et al. [34] to reduce the number of segments required for a given accuracy.

The ability to accurately compensate for off-resonance effects as demonstrated here may increase the feasibility of using other acquisition methods with long readout times, such as echovolume imaging [46].

Although this paper has focused on MR image reconstruction in the presence of field inhomogeneities, the general approach is also applicable to image reconstruction with compensation for other sources of undesired (but known) spin phase accrual, such as eddy currents and concomitant gradient effects [47], [48]. An iterative method based on an explicit signal model like (1) should yield more accurate images compared with conventional approaches to compensating for such effects.

We have ignored spin-spin relaxation during the signal readout in our signal model (1). However, many aspects of the algorithms we have described are also applicable to problems where both spin density and spin relaxation are estimated from multiecho measurements [9], [16], [49], [50]. The framework for the min-max time interpolation provided by (16) can be extended to include relaxation effects, such as $R_{2}^{*}$. The simplifications that resulted in (18) are not available in this case and computation of the interpolator may be more expensive. Preliminary testing shows that the high accuracy of the time segmentation method can still be achieved without knowing the exact field and $R_{2}^{*}$ maps. This work will be included in a future paper.

\section{ACKNOWLEDGMENT}

The authors would also like to thank V. Olafsson for his input and careful reading. 


\section{REFERENCES}

[1] K. Sekihara, M. Kuroda, and H. Kohno, "Image restoration from nonuniform magnetic field influence for direct Fourier NMR imaging," Phys. Med. Biol., vol. 29, no. 1, pp. 15-24, 1984.

[2] E. Yudilevich and H. Stark, "Spiral sampling in magnetic resonance imaging — The effect of inhomogeneities," IEEE Trans. Med. Imag., vol. MI-6, no. 4, pp. 337-345, 1987.

[3] D. C. Noll, C. H. Meyer, J. M. Pauly, D. G. Nishimura, and A. Macovski, "A homogeneity correction method for magnetic resonance imaging with time-varying gradients," IEEE Trans. Med. Imag., vol. 10, pp. 629-637, Dec. 1991.

[4] D. C. Noll, J. M. Pauly, C. H. Meyer, D. G. Nishimura, and A. Macovski, "Deblurring for non-2D Fourier transform magnetic resonance imaging," Magn. Reson. Med., vol. 25, pp. 319-333, 1992.

[5] Y. M. Kadah and X. Hu, "Simulated phase evolution rewinding (SPHERE): A technique for reducing $B_{0}$ inhomogeneity effects in MR images," Magn. Reson. Med., vol. 38, pp. 615-627, 1997.

[6] H. Schomberg, "Off-resonance correction of MR images," IEEE Trans. Med. Imag., vol. 18, pp. 481-495, June 1999.

[7] L.-C. Man, J. M. Pauly, and A. Macovski, "Multifrequency interpolation for fast off-resonance correction," Magn. Reson. Med., vol. 37, pp. 785-792, 1997.

[8] M. S. NessAiver, "Optical implementation of spiral EPI field homogeneity correction and the decoupled automated rotation and translation algorithm (DFT-DART)," in Proc. ISMRM 8th Scientific Meeting, 2000, p. 111.

[9] B. P. Sutton, D. C. Noll, and J. A. Fessler, "Simultaneous estimation of image and inhomogeneity field map," in Proc. ISMRM Workshop Min. MR Data Acquisition Methods: Making More with Less, 2001, pp. $15-18$.

[10] D. C. Noll, "Rapid MR image acquisition in the presence of background gradients," in Proc. IEEE Int. Symp. Biomed. Imaging, 2002, pp. $725-728$.

[11] — , "Multishot Rosette trajectories for spectrally selective MR imaging," IEEE Trans. Med. Imag., vol. 16, pp. 372-377, Aug. 1997.

[12] P. Munger, G. R. Crelier, T. M. Peters, and G. B. Pike, "An inverse problem approach to the correction of distortion in EPI images," IEEE Trans. Med. Imag., vol. 19, pp. 681-689, July 2000.

[13] L.-C. Man, J. M. Pauly, and A. Macovski, "Correcting severe local inhomogeneity blurs by spatially variant deconvolution," in Proc. SMR $3 r d$ Scientific Meeting, vol. 2, 1995, p. 738.

[14] T. B. Harshbarger and D. B. Twieg, "Iterative reconstruction of single-shot spiral MRI with off resonance," IEEE Trans. Med. Imag., vol. 18, pp. 196-205, Mar. 1999.

[15] K. P. Pruessmann, M. Weiger, M. B. Scheidegger, and P. Boesiger, "SENSE: Sensitivity encoding for fast MRI," Magn. Reson. Med., vol. 42, pp. 952-962, 1999.

[16] B. Sutton, S. J. Peltier, J. A. Fessler, and D. Noll, "Simultaneous estimation of $I_{o}, R_{2}^{*}$, and field map using a multi-echo spiral acquisition," in Proc. ISMRM 10th Scientific Meeting, 2002, p. 1323.

[17] A. Dutt and V. Rokhlin, "Fast Fourier transforms for nonequispaced data," SIAM J Sci. Comput., vol. 14, no. 6, pp. 1368-1393, 1993.

[18] J. A. Fessler and B. P. Sutton, "Nonuniform fast Fourier transforms using min-max interpolation," IEEE Trans. Signal Processing, vol. 51, pp. 560-574, Feb. 2003.

[19] — " "A min-max approach to the multidimensional nonuniform FFT: Application to tomographic image reconstruction," in Proc. IEEE Intl. Conf. Image Processing, vol. 1, 2001, pp. 706-709.

[20] S. A. R. Kannengießer, J. M. Pauly, and R. K. Butts, "Fast image reconstruction for sensitivity encoded spiral imaging," in Proc. ISMRM 9th Scientific Meeting, 2001, p. 1800.

[21] B. Sutton, J. A. Fessler, and D. Noll, "A min-max approach to the nonuniform $\mathrm{N}$-dimensional FFT for rapid iterative reconstruction of MR images," in Proc. ISMRM 9th Scientific Meeting, 2001, p. 763.

[22] G. Beylkin, "On the fast Fourier transform of functions with singularities," Appl. Computat. Harmonic Anal., vol. 2, pp. 363-381, 1995.

[23] T. Strohmer, "Computationally attractive reconstruction of bandlimited images from irregular samples," IEEE Trans. Image Processing, vol. 6, pp. 540-548, Apr. 1997.

[24] - "Numerical analysis of the nonuniform sampling problem," $J$. Comp. Appl. Math., vol. 122, no. 1-2, pp. 297-316, 2000.

[25] K. P. Pruessmann, M. Weiger, P. Börnert, and P. Boesiger, "Advances in sensitivity encoding with arbitrary k-space trajectories," Magn. Reson. Med., vol. 46, pp. 638-651, 2001.
[26] D. G. Nishimura, Principles of Magnetic Resonance Imaging. Stanford, CA: Dept. Elect. Eng., Stanford Univ., 1996.

[27] J. I. Jackson, C. H. Meyer, D. G. Nishimura, and A. Macovski, "Selection of a convolution function for Fourier inversion using gridding," IEEE Trans. Med. Imag., vol. 10, pp. 473-478, Sept. 1991.

[28] R. Van de Walle, H. H. Barrett, K. J. Myers, M. I. Altbach, B. Desplanques, A. F. Gmitro, J. Cornelis, and I. Lemahieu, "Reconstruction of MR images from data acquired on a general nonregular grid by pseudoinverse calculation," IEEE Trans. Med. Imag., vol. 19, pp. 1160-1167, Dec. 2000.

[29] E. M. Haacke, R. W. Brown, M. R. Thompson, and R. Venkatesan, Magnetic Resonance Imaging: Physical Principles and Sequence Design. New York, 1999: John Wiley and Sons.

[30] J. A. Fessler and W. L. Rogers, "Spatial resolution properties of penalized-likelihood image reconstruction methods: Space-invariant tomographs," IEEE Trans. Image Processing, vol. 5, pp. 1346-1358, Sept. 1996.

[31] P. J. Huber, Robust Statistics. New York: Wiley, 1981.

[32] Y.-H. Kao and J. R. MacFall, "Correction of MR k-space data corrupted by spike noise," IEEE Trans. Med. Imag., vol. 19, pp. 671-680, July 2000

[33] D. C. Noll, "Reconstruction techniques for magnetic resonance imaging," Ph.D. dissertation, Stanford Univ., Stanford, CA, 1991.

[34] P. Irarrazabal, C. H. Meyer, D. G. Nishimura, and A. Macovski, "Inhomogeneity correction using an estimated linear field map," Magn. Reson. Med., vol. 35, pp. 278-282, 1996.

[35] H. Sedarat and D. G. Nishimura, "On the optimality of the gridding reconstruction algorithm," IEEE Trans. Med. Imag., vol. 19, pp. 306-317, Apr. 2000.

[36] J. G. Pipe, "Reconstructing MR images from undersampled data: Dataweighting considerations," Magn. Reson. Med., vol. 43, pp. 867-875, 2000.

[37] J. G. Pipe and P. Menon, "Sampling density compensation in MRI: Rationale and an iterative numerical solution," Magn. Reson. Med., vol. 41, pp. 179-186, 1999.

[38] Y. Qian, J. Lin, and D. Jin, "Reconstruction of MR images from data acquired on an arbitrary k-space trajectory using the same-image weight," Magn. Reson. Med., vol. 48, pp. 306-311, 2002.

[39] H. Schomberg, "Notes on direct and gridding-based Fourier inversion methods," in Proc. IEEE Int. Symp. Biomed. Imaging, 2002, pp. 645-648.

[40] Y. M. Kadah and X. Hu, "Algebraic reconstruction for magnetic resonance imaging under $B_{0}$ inhomogeneity," IEEE Trans. Med. Imag., vol. 17, pp. 362-370, June 1998.

[41] N. H. Clinthorne, T.-S. Pan, P.-C. Chiao, W. L. Rogers, and J. A. Stamos, "Preconditioning methods for improved convergence rates in iterative reconstructions," IEEE Trans. Med. Imag., vol. 12, pp. 78-83, Mar. 1993.

[42] J. A. Fessler and S. D. Booth, "Conjugate-gradient preconditioning methods for shift-variant PET image reconstruction," IEEE Trans. Image Processing, vol. 8, pp. 688-699, May 1999.

[43] E. Schneider and G. Glover, "Rapid in vivo proton shimming," Magn. Reson. Med., vol. 18, pp. 335-347, 1991.

[44] B. Sutton, J. A. Fessler, and D. Noll, "Field-corrected imaging using joint estimation of image and field map," in Proc. ISMRM 10th Scientific Meeting, 2002, p. 737.

[45] — - "Iterative MR image reconstruction using sensitivity and inhomogeneity field maps," in Proc. ISMRM 9th Scientific Meeting, 2001, p. 771

[46] A. W. Song, E. C. Wong, and J. S. Hyde, "Echo-volume imaging," Magn. Reson. Med., vol. 32, pp. 668-671, 1994.

[47] M. A. Bernstein, X. J. Zhou, J. A. Polzin, K. F. King, A. Ganin, N. J. Pelc, and G. H. Glover, "Concomitant gradient terms in phase constrast MR: Analysis and correction," Magn. Reson. Med., vol. 39, pp. 300-308, 1998.

[48] K. F. King, A. Ganin, X. J. Zhou, and M. A. Bernstein, "Concomitant gradient field effects in spiral scans," Magn. Reson. Med., vol. 41, pp. 103-112, 1999.

[49] M. I. Miller, T. J. Schaewe, C. S. Bosch, and J. J. H. Ackerman, "Model-based maximum likelihood estimation for phase- and frequency-encoded magnetic-resonance-imaging data," J. Magn. Reson., ser. B, vol. 107, pp. 210-221, 1995.

[50] S. J. Peltier, B. Sutton, J. A. Fessler, and D. Noll, "Simultaneous estimation of $I_{o}, R_{2}^{*}$, and field map using a multi-echo spiral acquisition," Hum. Brain Mapping, p. 10081, 2002. 